\title{
Influence of laser energy on the electron temperature of a laser-induced Mg plasma
}

\author{
Emmanuel Asamoah ${ }^{1}$ Yao Hongbing ${ }^{1}$
}

Received: 6 October 2016 / Accepted: 7 December 2016 / Published online: 23 December 2016

(c) The Author(s) 2016. This article is published with open access at Springerlink.com

\begin{abstract}
The magnesium plasma induced by a $1064-\mathrm{nm}$ Q-switched Nd:YAG laser in atmospheric air was investigated. The evolution of the plasma was studied by acquiring spectral images at different laser energies and delay times. We observed that the intensities of the spectral lines decrease with larger delay times. The electron temperature was determined using the Boltzmann plot method. At a delay time of $100 \mathrm{~ns}$ and laser energy of $350 \mathrm{~mJ}$, the electron temperature attained their highest value at $10164 \mathrm{~K}$ and then decreases slowly up to $8833.6 \mathrm{~K}$ at $500 \mathrm{~ns}$. We found that the electron temperature of the magnesium plasma increases rapidly with increasing laser energy.
\end{abstract}

\section{Introduction}

In the past decades, there has been an increasing interest in laser-induced breakdown spectroscopy (LIBS) in several research fields such as material processing, space applications and diagnostic techniques $[1,2]$. LIBS is an essential analytical technique for identification of the elemental composition of a sample [3-6].

In this technique, a highly energetic laser pulses generated from a Q-switched laser source is focused through a suitable focusing lens to generate a plume of plasma rich in atoms, electrons and ions on the surface of the sample. The focused beam results in the evaporation, atomization and ionization of the sample surface material, and the emitted

Emmanuel Asamoah

asamoah_grace@hotmail.com

1 School of Mechanical Engineering, Jiangsu University, Zhenjiang 212013, Jiangsu, China radiations in this process are used for the identification of the elemental composition of the sample [7-9].

LIBS offers many advantages over other spectroscopic techniques such as mass spectroscopy, electrode spark and inductively coupled plasma. Some of LIBS unique advantages include the ability to make in situ analysis even in hostile environment, remote sensing, little or no preparation of the sample, and multi-element analysis [10-12]. Based on these advantages, LIBS has been extensively investigated on several samples including liquids [13], gases [14] and as well from non-conducting and conducting solids [15].

LIBS is also a spectroscopic technique for the determination of the density of electron, electron temperature and number densities of the plasma plume $[16,17]$. Laserinduced plasma temperatures are influenced by various experimental conditions such as ambient surrounding, laser energy and laser pulse width. Most of the studies on LIBS temperatures report that the plasma temperature tends to increase with increasing laser energy, pulse width, laser wavelength and ambient pressure [18-21].

Although LIBS has been employed successfully in several experimental and theoretical works, to analyze the spectroscopic composition of the samples, there are still many aspects which need to be investigated [6]. For example, Barthelemy et al. [22] investigated the influence of the laser parameters of an aluminum-induced plasma. They reported that the temperature slightly decreases at the plasma edge and close to the aluminum surface. This decrease in temperature at the plasma edge is basically caused by radiative cooling, while the decrease in temperature at the aluminum surface is as a result of thermal conduction. The authors also found that at the first microsecond of the ablation plume, the electron density was spatially homogeneous.

Zhang et al. [23] also reported on the influence of laser parameters on plasma temperature. Their results predicted 
a general trend that LIP temperature increases with increasing laser parameters.

In this work, we shall report on laser-induced magnesium plasma with different laser energies and several time delays in order to optimize the spectroscopic performance, and investigate the influence of laser energy on the electron temperature. Section 2 of this work is devoted to the experimental setup and procedure used to obtain the timeresolved emission spectra and the temporal evolution of the electron temperature. The experimental results are presented in Sect. 3. Finally, Sect. 4 gives the conclusion of the results of this work.

\section{Experimental setup}

The experimental setup of the laser-induced breakdown spectroscopy is shown in Fig. 1. The setup consists of Nd:YAG laser source for plasma formation and vaporization of the target, monochromator and a time-resolved detector. The 1064-nm beam from a Q-switched Nd:YAG laser, with $10 \mathrm{~ns}$ pulse width and $1-\mathrm{Hz}$ pulse repetition rate (PRR), emitting a laser pulse with energy of 150, 250 and $350 \mathrm{~mJ}$, which was measured by a pyrometric detector.

The laser beam was focused horizontally onto the target surface by a means of a quartz lens with $150 \mathrm{~mm}$ focal length. The distance from the lens to the target was set to about $14 \mathrm{~cm}$ in order to gain a nearly hemispherical plasma plume and a reproducible breakdown. The plasma was generated in air at atmospheric pressure on $\sim 1-\mathrm{mm}^{2}$ surface area of the metal target. The content purity of the target was almost $99.99 \%$ magnesium plate, which was previously well polished before exposing to the laser beam.

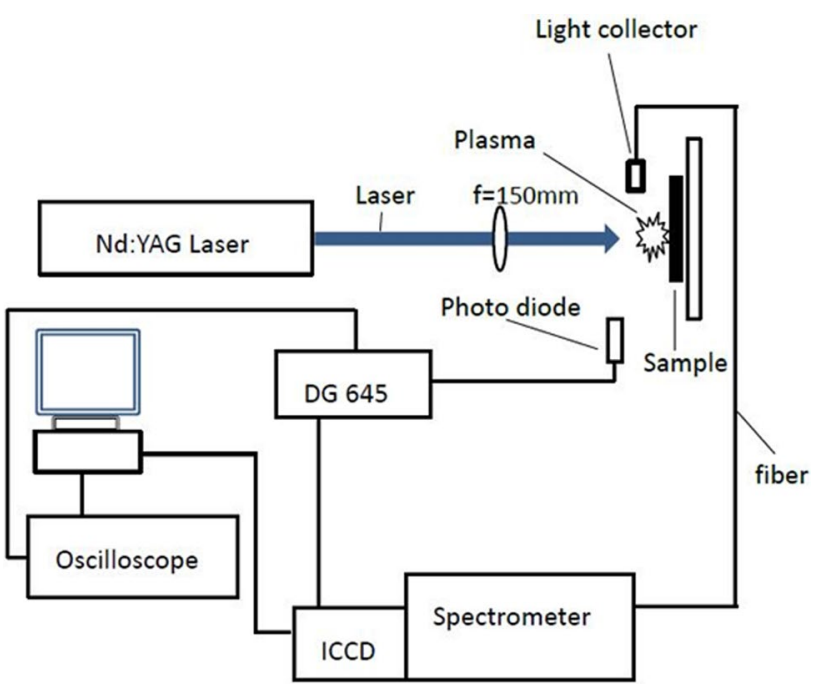

Fig. 1 Schematic diagram of the experimental setup for laserinduced breakdown spectroscopy
The plasma emission was collected by a fiber bundle, which is coupled to a Mechelle spectrometer built with a gateable intensified charge-coupled device (ICCD) camera with a $1024 \times 1024$ pixels (istar from Andor Technology). The ICCD detector was synchronized with the trigger of the laser pulse. The synchronization was done using a digital generator (Stanford Research System, model DG645) and the oscilloscope (YOKOGAWA, digital oscilloscope DL9140, Japan). The gate delay was also monitored with the oscilloscope, which was triggered by signals collected by the photodiode. The time delay of the ICCD was varied from 100 to $1000 \mathrm{~ns}$ of the different laser energies for the spectra detection. In each measurement, the acquisitions were integrated over a suitable number of accumulations, in order to increase the signal-to-noise ratio. The atomic
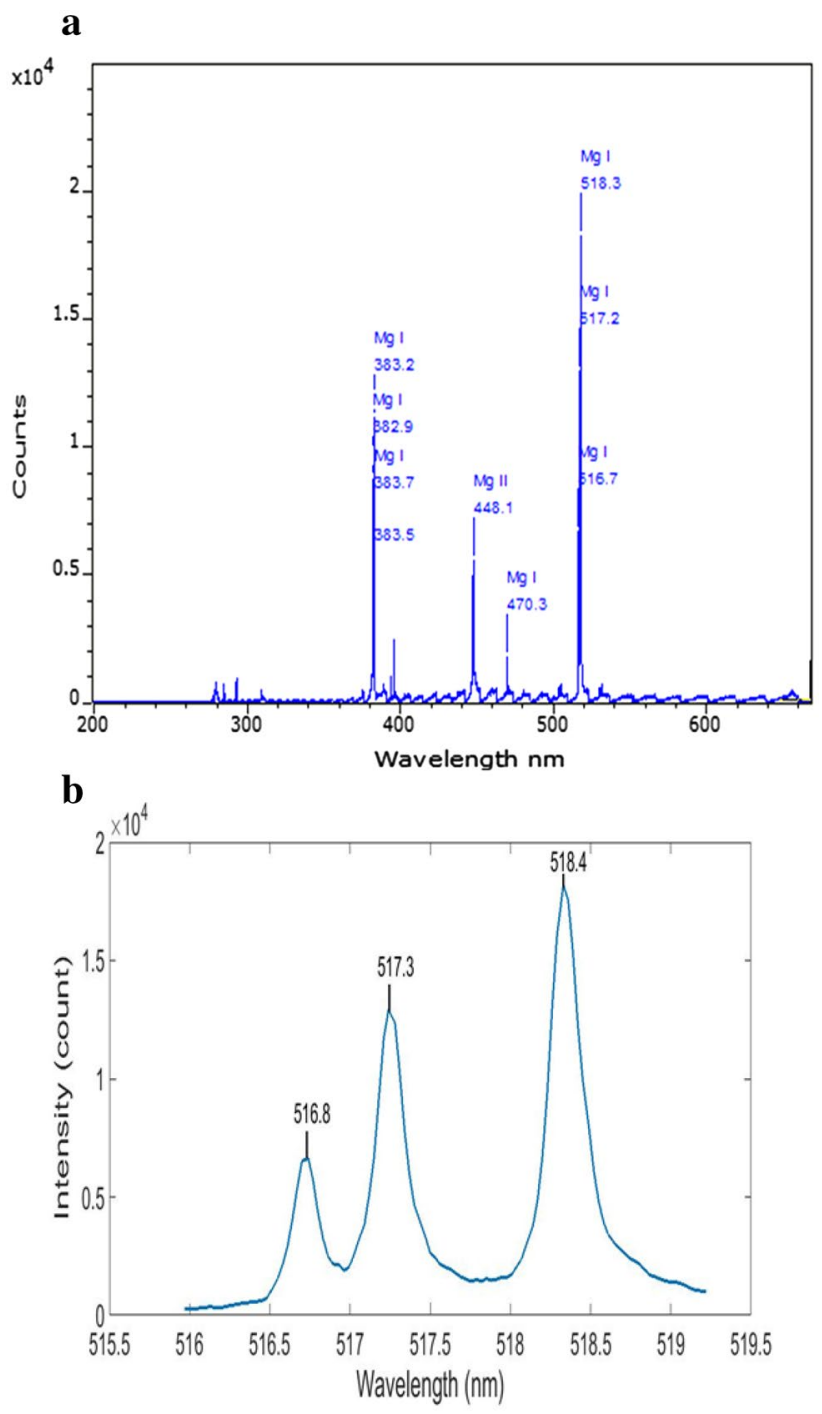

Fig. 2 a LIBS spectra of laser-induced magnesium plasma at a delay time of $100 \mathrm{~ns}$ and laser energy of $350 \mathrm{~mJ}$. b LIBS spectra of laserinduced magnesium plasma at a delay time of $100 \mathrm{~ns}$ and laser energy of $350 \mathrm{~mJ}$ in the spectral region of $515.97-519.22 \mathrm{~nm}$ 
emission lines were identified using available atomic spectra data compiled in the national institute for standards and technology database [24].

\section{Results and discussion}

\subsection{The spectra of the magnesium-induced plasma}

The emission spectrum of the laser-induced magnesium plasma is shown in Fig. 2. The plasma emission was collected by a fiber bundle, which is coupled to a Mechelle spectrometer built with an intensified charge-coupled device (ICCD). The spectra were taken at a delay time of $100 \mathrm{~ns}$ and pulse energy of $350 \mathrm{~mJ}$. The characteristic intensity of the $\mathrm{Mg}$ emission rapidly decreases with a larger delay time, due to bremsstrahlung emission and radiative recombination between the ions and electrons [25], and

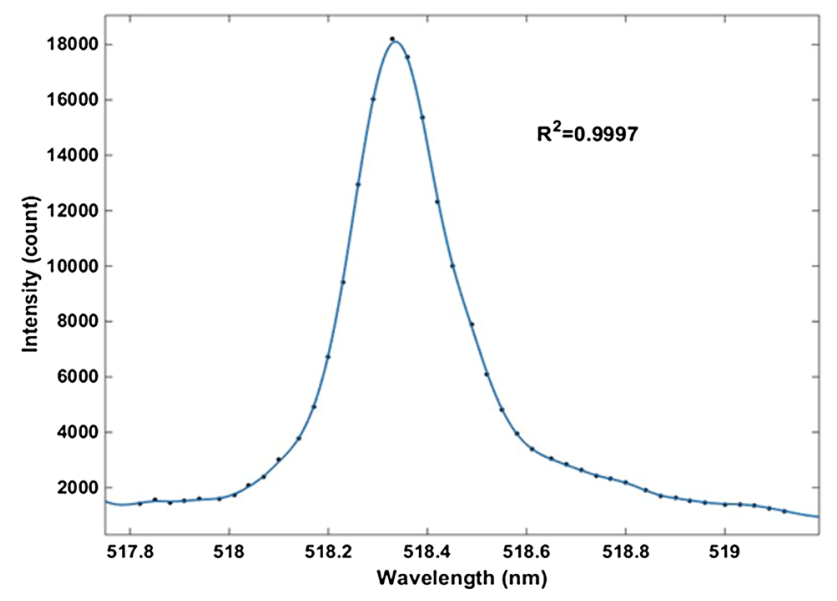

Fig. 3 Lorentz fit of MgI 518.4-nm spectral line at a delay of $100 \mathrm{~ns}$ and laser energy of $350 \mathrm{~mJ}$ this is as a result of plasma quenching. At a delay time of $100 \mathrm{~ns}$, a strong continuum is observed. The MgI 518.4-nm spectral line reaches the highest intensity with laser energy of $350 \mathrm{~mJ}$, and this is fitted into the Lorentz profile in Fig. 3. In order to estimate the electron temperature of the laser-induced magnesium plasma, we study different emission spectra at several delay times. Figure 4 depicts a timeresolved emission spectrum of laser-induced magnesium plasma at different delay times.

\subsection{The local thermodynamic equilibrium and the optical thinness of the plasma}

The determination of the plasma temperature by the Boltzmann plot method requires the plasma to be in the state of thermodynamic equilibrium (LTE) and also must be optically thin. The Mg-induced plasma was optically thin for the line intensities used in the determination of the plasma temperatures [26]. This was observed from the peak intensities of the ratio of the $\mathrm{Mg}$ lines used. The experimental data and the stimulation validate that the local thermodynamic equilibrium condition is satisfied with plasmas of longer delay times. This depends on the plasma parameters such as the ambient gas, laser energy and pulse duration used in the experiments $[27,28]$. The most commonly used method to warrant the existence of LTE is the electron energy distribution function (EEDF) Maxwell distribution, and the McWhirter criterion [23, 29-31]. The McWhirter criterion defines the minimum electron number density which must be present in order for LTE to exist, which can be expressed mathematically as [3]:

$N_{e} \geq 1.6 \times 10^{12} T^{1 / 2}\left(\Delta E_{m n}\right)^{3}$

where $N_{e}$ is the electron density $\left(\mathrm{cm}^{-3}\right), T$ is the plasma temperature $(\mathrm{K})$ and $\Delta E_{m n}$ is the higher energy difference $(\mathrm{eV})$ between the upper and lower energy states of the Mg lines used.
Fig. 4 Time-resolved emission spectra of a laser-induced $\mathrm{Mg}$ plasma at different delay times, in the spectra region of $515.97-519.22 \mathrm{~nm}$ with a laser energy of $350 \mathrm{~mJ}$

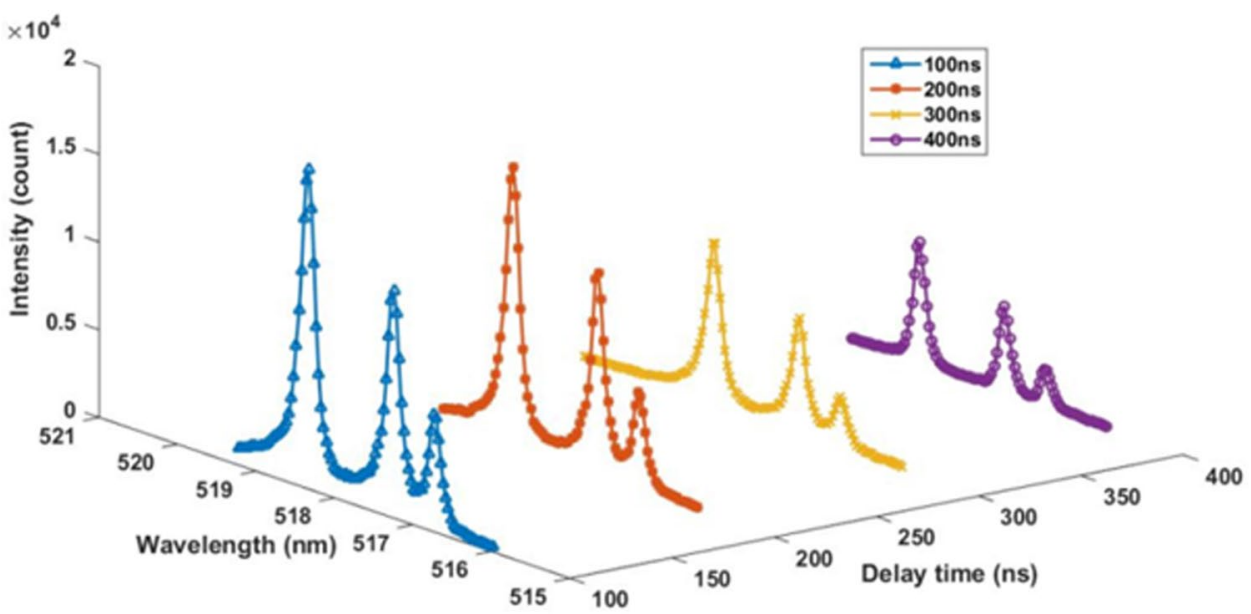


Research recently has revealed that the McWhirter criterion is necessary but insufficient to assess the existence of LTE [32], since it is derived for stationary and homogenous plasmas. From this work, we established that the LTE is satisfied because the electron density deduced from the stark broadening is higher than $10^{16} \mathrm{~cm}^{-3}$.

\subsection{Theoretical model of the electron temperature}

Assuming plasma in LTE, the population of the energy levels of the species obeys the Boltzmann's distribution law $[17,23]$, which can be given by the expression:

$N_{m}=N \frac{g_{m} e \frac{E_{m}}{k T}}{U(T)}$

where the subscript $m$ stands for the upper energy level of the transition, $N_{m}$ and $g_{m}$ are the population $\left(\mathrm{m}^{-3}\right)$ of the energy levels and the statistical weight of the upper energy level, respectively, $E_{m}$ is the energy $(\mathrm{eV})$ of the upper energy level, $N$ is the number density $\left(\mathrm{m}^{-3}\right), k$ is the Boltzmann's constant $\left(\mathrm{eV} \mathrm{k}^{-1}\right), T$ is the electron temperature $(\mathrm{k})$ and $U(T)$ is the partition function. The emissivity $\varepsilon_{m n}\left(\mathrm{~W} \mathrm{~m}^{-3}\right)$, of a particular transition of the species, can be written in the form:

$\varepsilon_{m n}=\frac{h c}{\lambda_{m n}} A_{m n} N_{m}$

where the subscript $n$ represents the lower energy level of the transition, $c$ is the speed of light $\left(\mathrm{m} \mathrm{s}^{-1}\right), h$ is the Planck's constant (Js), $A_{m n}$ is the transition probability $\left(\mathrm{s}^{-1}\right)$ and $\lambda_{m n}$ is the transition wavelength (m). From the expression of the emissivity, substituting the population of the energy levels $N_{m}$ into Eq. (2), we will obtain:

$\varepsilon_{m n}=\frac{h c}{\lambda_{m n}} A_{m n} N \frac{g_{m} e \frac{E_{m}}{k T}}{U(T)}$

For experimental systems, we can replace the emissivity by the line intensity, and by taking the natural logarithm of Eq. (4), we obtain the expression in the form:

$\ln \left(\frac{I_{m n} \lambda_{m n}}{A_{m n} g_{m n}}\right)=-\frac{E_{m}}{k T}+\ln \left(\frac{h c N}{U(T)}\right)$

By plotting the magnitude on the left side against the energy of the upper level in Eq. (5), yields a linear fit with a slope of $-1 / k T$. The electron temperature can be calculated from the slope of the Boltzmann's plot without knowing the partition function. The magnesium atomic lines at wavelength of $383.2,470.3$ and $518.4 \mathrm{~nm}$ were used to calculate the electron temperature. The spectroscopic data of $A_{m n}, E_{m}$ and $g_{m}$ of the $\mathrm{MgI}$ spectral line can be retrieved from the NIST database. The spectroscopic data of the $\mathrm{MgI}$ spectra lines are listed in Table 1.
The Boltzmann plot at a delay time of $100 \mathrm{~ns}$ and energy of $100 \mathrm{~mJ}$ is shown in Fig. 5, and the slope of the plot yielded an electron temperature of $8810 \mathrm{~K}$. The temporal evolution of the electron temperature is shown in Fig. 6, with a delay time varying from 100 to $1000 \mathrm{~ns}$ at three

Table 1 The spectroscopic data of $\mathrm{Mg}$ I and $\mathrm{Mg}$ II lines used, from the NIST database [24]

\begin{tabular}{lllll}
\hline Spectra line & $\lambda_{m n}(\mathrm{~nm})$ & $E_{m}(\mathrm{eV})$ & $A_{m n}\left(\times 10^{8} \mathrm{~s}^{-1}\right)$ & $g_{m}$ \\
\hline $\mathrm{MgI}$ & 383.0 & 5.95 & 0.90 & 3 \\
$\mathrm{MgI}$ & 383.2 & 5.95 & 1.21 & 5 \\
$\mathrm{MgI}$ & 470.3 & 6.98 & 0.22 & 5 \\
$\mathrm{MgI}$ & 516.8 & 5.11 & 0.11 & 3 \\
$\mathrm{MgI}$ & 517.3 & 5.11 & 0.34 & 3 \\
$\mathrm{MgI}$ & 518.4 & 5.11 & 0.56 & 3 \\
$\mathrm{MgII}$ & 448.1 & 11.63 & 2.33 & 8 \\
\hline
\end{tabular}

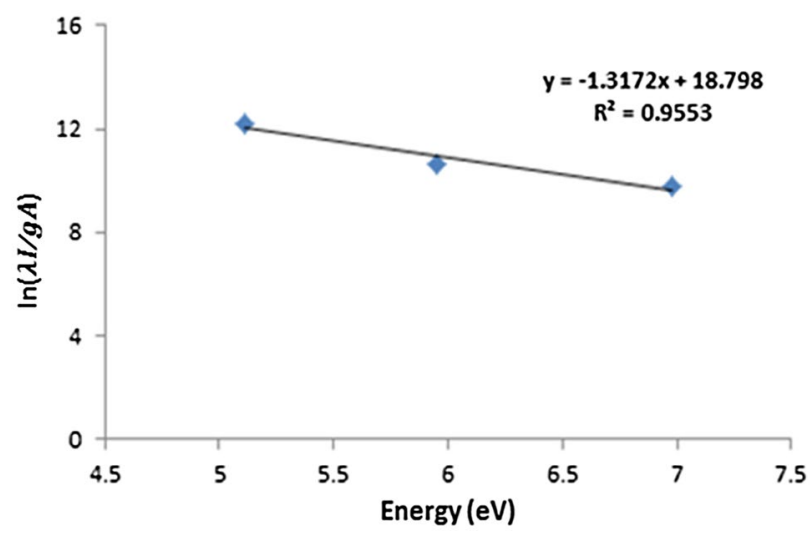

Fig. 5 Boltzmann plot obtained from $\mathrm{MgI}$ lines at a delay time of $100 \mathrm{~ns}$ and laser energy of $100 \mathrm{~ns}$, using the spectral data of $\mathrm{MgI}$ 383.2, $\mathrm{MgI} 470.3$ and $\mathrm{MgI} 518.4 \mathrm{~nm}$

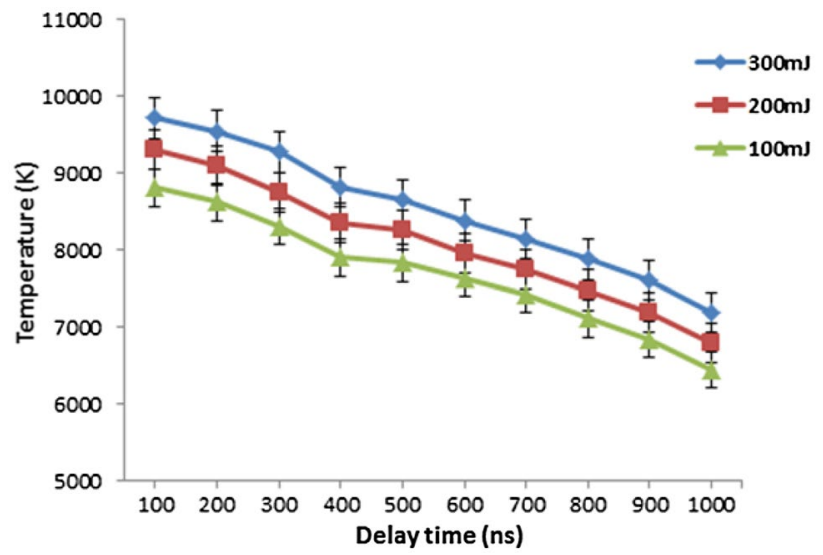

Fig. 6 Temporal evolution of the electron temperature of the magnesium-induced plasma, with laser energies of 100, 200 and $300 \mathrm{~mJ}$ 


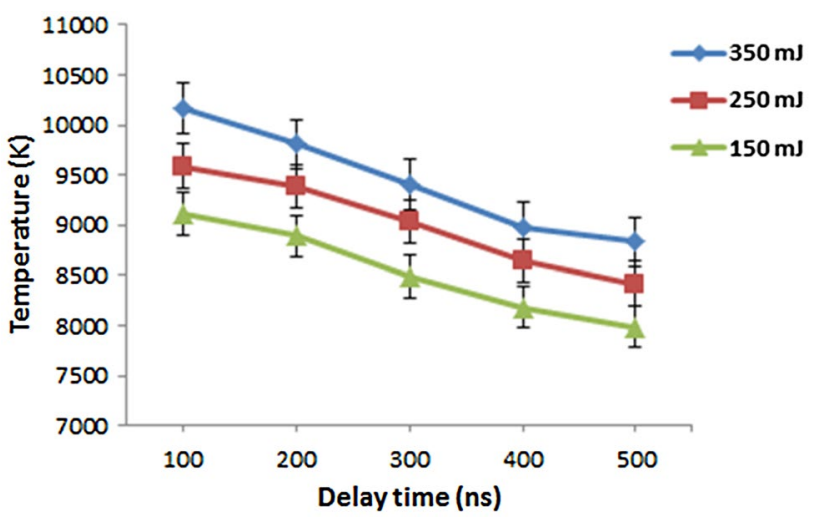

Fig. 7 Temporal evolution of the electron temperature of the magnesium-induced plasma, with laser energies of 150, 250 and $350 \mathrm{~mJ}$

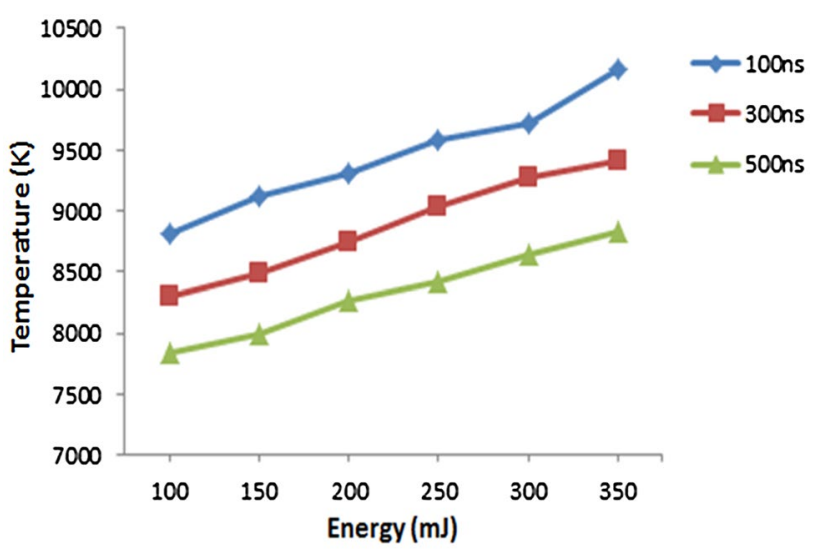

Fig. 8 Plot of the electron temperature against laser energy, at three different delay times, 100, 300 and $500 \mathrm{~ns}$

different laser energies. The error bars depicted the standard deviations in determining the value of the electron temperature. In Fig. 7, the electron temperature reaches their highest value at $10164 \mathrm{~K}$, with a delay time of $100 \mathrm{~ns}$ and laser energy of $350 \mathrm{~mJ}$, and then decreases slowly up to $8833.6 \mathrm{~K}$ at $500 \mathrm{~ns}$. Figure 8 depicts a plot of the electron temperature against the energy of the laser. As evident from the figure, the electron temperature increases with increasing laser energy.

\section{Conclusion}

The emission spectroscopy of the laser-induced magnesium plasma in atmospheric air was investigated. The emission spectral lines were resolved at different delay times. From the time-resolved emission spectra of the laser-induced Mg plasma, we observed that the characteristic intensities of the spectral lines rapidly decrease with a larger delay times, this was as a result of bremsstrahlung emission and radiative recombination between the ions and electrons, and this is due to plasma quenching. At a delay time of $100 \mathrm{~ns}$, a strong continuum was observed. The MgI 518.4-nm spectral line reaches the highest intensity with energy of $350 \mathrm{~mJ}$. The electron temperature was determined from the Boltzmann plot method, using the magnesium atomic lines at wavelength of $383.2,470.3$ and $518.4 \mathrm{~nm}$. A plot of $\ln (\lambda I / A)$ against $E_{m}$ gave a slope of $-1 / k T$. At a delay time of $100 \mathrm{~ns}$ and laser energy of $350 \mathrm{~mJ}$, the electron temperature attained their highest value at $10164 \mathrm{~K}$. The electron temperature then decreases slowly up to $8833.6 \mathrm{~K}$ at a delay time of $500 \mathrm{~ns}$. At laser energies of 100, 200 and $300 \mathrm{~mJ}$ at a delay time of $100 \mathrm{~ns}$, the electron temperatures were found to be 8810,9303 and $9724 \mathrm{~K}$, respectively. We found that the electron temperature of the Mg plasma increases rapidly with increasing laser energy.

Open Access This article is distributed under the terms of the Creative Commons Attribution 4.0 International License (http://creativecommons.org/licenses/by/4.0/), which permits unrestricted use, distribution, and reproduction in any medium, provided you give appropriate credit to the original author(s) and the source, provide a link to the Creative Commons license, and indicate if changes were made.

\section{References}

1. P.R. Willmott, J.R. Huber, Pulsed laser vaporization and deposition. Rev. Mod. Phys. 72, 315-328 (2000)

2. A. Giardini, V. Marotta, A. Morone, S. Orlando, G.P. Parisi, Thin films deposition in RF generated plasma by reactive pulsed laser ablation. Appl. Surf. Sci. 197, 338-342 (2002)

3. S.Z. Mortazavi, P. Parvin, M.R. Mousavi Pour, A. Reyhani, A. Moosakhani, S. Moradkhani, Time-resolved evolution of metal plasma induced by Q-switched Nd:YAG and ArF-excimer lasers. Opt. Laser Technol. 62, 32-39 (2014)

4. Aguilera, J.A., Aragón, C.: A comparison of the temperatures and electron densities of laser-produced plasmas obtained in air, argon, and helium at atmospheric pressure. Appl. Phys. A 69, S475-S478

5. S. Morel, N. Leone, P. Adam, J. Amouroux, Detection of bacteria by time-resolved laser-induced breakdown spectroscopy. Appl. Opt. 42, 6184-6191 (2003)

6. M. Capitelli, A. Casavola, G. Colonna, A. De Giacomo, Laserinduced plasma expansion: theoretical and experimental aspects. Spectrochim. Acta Part B Atomic Spectrosc. 59, 271-289 (2004)

7. Y. Li, H. Zhang, Z. Jiang, Z. Li, C. Hu, Spectroscopic characterization of aluminum plasma using laser-induced breakdown spectroscopy. Opt. Int. J. Light Electron Opt. 125, 2851-2855 (2014)

8. R.T. Wainner, R.S. Harmon, A.W. Miziolek, K.L. McNesby, P.D. French, Analysis of environmental lead contamination: comparison of LIBS field and laboratory instruments. Spectrochim. Acta Part B 56, 777-793 (2001)

9. W.F. Luo, X.X. Zhao, Q.B. Sun, C.X. Gao, J. Tang, H.J. Wang, W. Zhao, Characteristics of the aluminum alloy plasma produced by a $1064 \mathrm{~nm} \mathrm{Nd}$ laser with different irradiances. Pramana 74, 945-959 (2010) 
10. M.E. Asgill, S. Groh, K. Niemax, D.W. Hahn, The use of multielement aerosol particles for determining temporal variations in temperature and electron density in laser-induced plasmas in support of quantitative laser-induced breakdown spectroscopy. Spectrochim. Acta Part B 109, 1-7 (2015)

11. M. Corsi, G. Cristoforetti, M. Giuffrida, M. Hidalgo, S. Legnaioli, V. Palleschi, A. Salvetti, E. Tognoni, C. Vallebona, Threedimensional analysis of laser induced plasmas in single and double pulse configuration. Spectrochim. Acta Part B 59, 723-735 (2004)

12. Z. Wang, T.-B. Yuan, Z.-Y. Hou, W.-D. Zhou, J.-D. Lu, H.-B. Ding, X.-Y. Zeng, Laser-induced breakdown spectroscopy in China. Front. Phys. 9, 419-438 (2014)

13. A.P.M. Michel, M. Lawrence-Snyder, S.M. Angel, A.D. Chave, Laser-induced breakdown spectroscopy of bulk aqueous solutions at oceanic pressures: evaluation of key measurement parameters. Appl. Opt. 46, 2507-2515 (2007)

14. V. Sturm, R. Noll, Laser-induced breakdown spectroscopy of gas mixtures of air, $\mathrm{CO}_{2}, \mathrm{~N}_{2}$, and $\mathrm{C}_{3} \mathrm{H}_{8}$ for simultaneous $\mathrm{C}, \mathrm{H}, \mathrm{O}$, and $\mathrm{N}$ measurement. Appl. Opt. 42, 6221-6225 (2003)

15. B. Le Drogoff, J. Margot, M. Chaker, M. Sabsabi, O. Barthélemy, T.W. Johnston, S. Laville, F. Vidal, Y. von Kaenel, Temporal characterization of femtosecond laser pulses induced plasma for spectrochemical analysis of aluminum alloys. Spectrochim. Acta Part B 56, 987-1002 (2001)

16. A. Arshad, S. Bashir, A. Hayat, M. Akram, A. Khalid, N. Yaseen, Q.S. Ahmad, Effect of magnetic field on laser-induced breakdown spectroscopy of graphite plasma. Appl. Phys. B 122, 1-10 (2016)

17. J.A. Aguilera, C. Aragón, Characterization of a laser-induced plasma by spatially resolved spectroscopy of neutral atom and ion emissions.: comparison of local and spatially integrated measurements. Spectrochim. Acta Part B 59, 1861-1876 (2004)

18. S. Bashir, N. Farid, K. Mahmood, M. Shahid Rafique, Influence of ambient gas and its pressure on the laser-induced breakdown spectroscopy and the surface morphology of laser-ablated $\mathrm{Cd}$. Appl. Phys. A 107, 203-212 (2012)

19. S.S. Harilal, C.V. Bindhu, R.C. Issac, V.P.N. Nampoori, C.P.G. Vallabhan, Electron density and temperature measurements in a laser produced carbon plasma. J. Appl. Phys. 82, 2140-2146 (1997)

20. S. Hafeez, N.M. Shaikh, B. Rashid, M.A. Baig, Plasma properties of laser-ablated strontium target. J. Appl. Phys. 103, 083117 (2008)
21. B. Rashid, S. Hafeez, N.M. Shaikh, M. Saleem, R. Ali, M.A. Baig, Diagnostics of copper plasma produced by the fundamental, second and third harmonics of a Nd:YAG laser. Int. J. Mod. Phys. B 21, 2697-2710 (2007)

22. O. Barthélemy, J. Margot, M. Chaker, M. Sabsabi, F. Vidal, T.W. Johnston, S. Laville, B. Le Drogoff, Influence of the laser parameters on the space and time characteristics of an aluminum laserinduced plasma. Spectrochim. Acta Part B 60, 905-914 (2005)

23. S. Zhang, X. Wang, M. He, Y. Jiang, B. Zhang, W. Hang, B. Huang, Laser-induced plasma temperature. Spectrochim. Acta Part B 97, 13-33 (2014)

24. NIST Atomic spectra database. http://Physics.nist.gov/PhysRefData/ASD/lines_form.html

25. J.J. Camacho, L. Diaz, S. Martinez-Ramirez, J.O. Caceres, Timeand space-resolved spectroscopic characterization of laserinduced swine muscle tissue plasma. Spectrochim. Acta Part B 111, 92-101 (2015)

26. I.Y. Elnasharty, Study on the influence of laser pulse duration in the long nanosecond regime on the laser induced plasma spectroscopy. Spectrochim. Acta Part B 124, 1-15 (2016)

27. H.-Y. Moon, B.W. Smith, N. Omenetto, Temporal behavior of line-to-continuum ratios and ion fractions as a means of assessing thermodynamic equilibrium in laser-induced breakdown spectroscopy. Chem. Phys. 398, 221-227 (2012)

28. S.Z. Shoursheini, B. Sajad, P. Parvin, Determination of gold fineness by laser induced breakdown spectroscopy with the simultaneous use of CW-CO ${ }_{2}$ and Q-SW Nd:YAG lasers. Opt. Lasers Eng. 48, 89-95 (2010)

29. J.B. Simeonsson, A.W. Miziolek, Spectroscopic studies of laserproduced plasmas formed in $\mathrm{CO}$ and $\mathrm{CO}_{2}$ using 193, 266, 355, 532 and, $1064 \mathrm{~nm}$ laser radiation. Appl. Phys. B 59(1), 1-9 (1994)

30. H.R. Griem, Validity of local thermal equilibrium in plasma spectroscopy. Phys. Rev. 131, 1170-1176 (1963)

31. G. Cristoforetti, G. Lorenzetti, S. Legnaioli, V. Palleschi, Investigation on the role of air in the dynamical evolution and thermodynamic state of a laser-induced aluminium plasma by spatialand time-resolved spectroscopy. Spectrochim. Acta Part B 65, 787-796 (2010)

32. G. Cristoforetti, A. De Giacomo, M. Dell'Aglio, S. Legnaioli, E. Tognoni, V. Palleschi, N. Omenetto, Local Thermodynamic Equilibrium in Laser-Induced Breakdown Spectroscopy: beyond the McWhirter criterion. Spectrochim. Acta Part B 65, 86-95 (2010) 